\title{
Efficacy of Allopurinol in Cardiovascular Diseases: A Systematic Review and Meta-Analysis
}

\author{
Waqas Ullah $^{\mathrm{a}, \mathrm{e}}$, Shristi Khanal ${ }^{\mathrm{a}}$, Rozi Khan ${ }^{\mathrm{b}}$, Bikash Basyala, Samavia Munir ${ }^{\mathrm{a}}$, \\ Artem Minalyan ${ }^{\mathrm{a}}$, M. Chadi Alraies ${ }^{\mathrm{c}}$, David L. Fischman ${ }^{\mathrm{d}}$
}

\begin{abstract}
Background: Given current evidence, the use of allopurinol for the prevention of major cardiovascular events (acute cardiovascular syndrome (ACS) or cardiovascular mortality) in patients undergoing coronary artery bypass graft (CABG), after index ACS or heart failure remains unknown.
\end{abstract}

Methods: Multiple databases were queried to identify studies comparing the efficacy of allopurinol in patients undergoing CABG, after ACS or heart failure. The unadjusted odds ratio (OR) was calculated using a random effect model.

Results: A total of nine studies comprising 850 patients (allopurinol 480 , control 370) were identified. The pooled OR of periprocedural ACS (OR: 0.25, 95\% confidence interval (CI): $0.06-0.96, \mathrm{P}=0.05$ ) and cardiovascular mortality (OR: $0.22,95 \% \mathrm{CI}: 0.07-0.71, \mathrm{P}=$ 0.01 ) was significantly lower in patients receiving allopurinol during CABG compared to patients in the control group. The overall number needed to treat (NNT) to prevent one ACS event was 11 (95\% CI: 7 28), while the NNT to prevent one death was 24 (95\% CI: 13 - 247). By contrast, the odds of cardiovascular mortality in the allopurinol group were not significantly different from the control group in patients on long-term allopurinol after ACS or heart failure (OR: 0.33 , 95\% CI: $0.01-8.21, \mathrm{P}=0.50)$ and (OR: $1.12,95 \% \mathrm{CI}: 0.39-3.20, \mathrm{P}$ $=0.83$ ), respectively. Similarly, the use of allopurinol did not reduce the odds of recurrent ACS events at 2 years (OR: $0.32,95 \%$ CI: 0.03 $-3.18, \mathrm{P}=0.33)$.

Conclusions: Periprocedural use of allopurinol might be associated with a significant reduction in the odds of ACS and cardiovascular mortality in patients undergoing CABG. Allopurinol, however, offers no long-term benefits in terms of secondary prevention of ACS or mortality. Larger scale studies are needed to validate our findings.

Manuscript submitted April 8, 2020, accepted April 17, 2020

Published online June 3, 2020

\footnotetext{
${ }^{a}$ Abington Jefferson Health, Abington, PA, USA

${ }^{b}$ Medstar Union Memorial Hospital, Baltimore, MD, USA

'Detroit Medical Center, DMC Heart Hospital, Detroit, MI, USA

${ }^{\mathrm{d}}$ Thomas Jefferson University, Philadelphia, PA, USA

${ }^{\mathrm{e}}$ Corresponding Author: Waqas Ullah, Abington Jefferson Health, 1200 Old

York Road, Abington, PA 19001, USA. Email: Waqasullah.dr@gmail.com
}

doi: https://doi.org/10.14740/cr1066
Keywords: Allopurinol; ACS; CABG; Heart failure

\section{Introduction}

Approximately $50 \%$ of patients undergoing coronary artery bypass graft (CABG) surgery have periprocedural reperfusion injury resulting in a considerable depression of the early postsurgical cardiac function (myocardial stunning) [1]. PostCABG myocardial stunning was widely held to result from uncontrolled free radical activity and thought to be amenable to anti-free radical therapy [2]. Given this, almost half the CABG patients theoretically could benefit from the administration of free oxygen radical scavengers. However, some clinical studies have failed to demonstrate convincing evidence on the production of free radicals during revascularization procedures. The addition of locally active agents (anti-free radical) to the cardioplegic solutions (used during the $\mathrm{CABG}$ procedure) has yielded disappointing clinical outcomes [3, 4].

Several studies had found an increased level of systemic free radical activity during and after $\mathrm{CABG}$ procedures when the antioxidant defense mechanism of myocardium was thought to be considerably impaired $[3,5]$. It is, therefore, reasonable to speculate that circulating free radical toxins when antioxidant defenses are decreased produce myocardial stunning [5]. This hypothesis provides a logical background for the limited, yet controversial clinical benefits associated with the systemic administration of direct free radical scavenging agents such as allopurinol at times of myocardial ischemia $[6,7]$.

The proposed mechanisms of allopurinol include systemic reduction of free radical activity by inhibition of the xanthine oxidase (a superoxide-generating enzyme), suppressing the secretion of inflammatory mediators and increasing nitric oxide production [8]. The later could also potentially reduce the incidence of acute coronary syndrome (ACS), as was suggested by Huang et al [9]. These theoretical benefits have led to studies examining the utilization of allopurinol before CABG and for the secondary prevention of ACS. However, evidence of its efficacy is scarce and controversial. Our study aims to bring consensus on the clinical use of allopurinol in patients undergoing CABG or after an ACS.

\section{Materials and Methods}

A structured literature search of electronic databases, including 


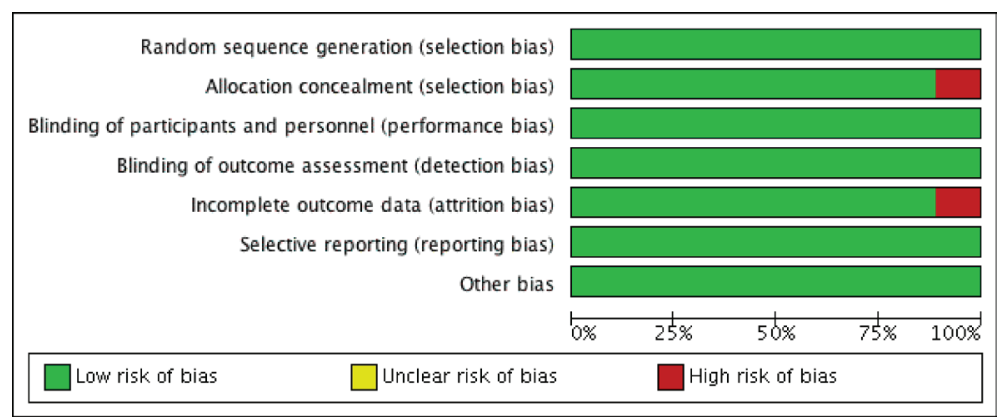

Figure 1. Summary of the methodological quality of the included studies showing minimal risk of bias (red).

MEDLINE (PubMed, Ovid), EMBASE, clinicaltrials.gov, and Cochrane, was performed using a combination of keywords and medical subject headings (MeSH). The keyword "allopurinol" was combined with a list of MeSH terms including "ischemic heart disease," "CAD," "stable angina," "myocardial infarction," "coronary artery disease," "MI," "heart failure," and "STEMI." Databases were last accessed on March 2, 2019. Based on our research question, articles from the reference lists relevant to the clinical question were also screened by an independent author (backward snowballing). All studies enrolling patients age greater than 18 years and reporting the role of allopurinol during $\mathrm{CABG}$, post-ACS or in heart failure were included. The primary efficacy endpoint was MI and cardiovascular mortality.

The statistical analysis was performed using the CochranMantel-Haenszel test under the random effect model to calculate pooled odds ratio (OR) for the endpoints. The estimated effect size was reported as a point estimate and $95 \%$ confidence interval (CI). An alpha criterion of $\mathrm{P}$ value less than 0.05 was considered statistically significant. If the eligibility of a study was dubious or influencing due to its large sample size, a sensitivity analysis was performed. Higgins I-squared $\left(\mathrm{I}^{2}\right)$ statistic model was used to evaluate variations in outcomes of included studies. $\mathrm{I}^{2}$ values of $50 \%$ or less corresponded to low to moderate, and $75 \%$ or higher indicated large amounts of heterogeneity. The publication bias was illustrated graphically with funnel plotting. All statistical analysis was performed using the Cochrane Review Manager version 5.3 .

\section{Quality assessment of the included studies}

The methodological quality was performed by screening all included articles for five different types of bias (selection, performance, detection, attrition, and reporting bias). The overall quality of the included studies was high (Fig. 1). All included clinical trials were randomized minimizing the risk of selection bias. Similarly, due to appropriate "allocation concealment" across most studies, the risk of sampling bias was low. Rashid et al [10] failed to obtain allocation concealment and in the study by Goicoechea et al [8], more than $10 \%$ of patients were lost at follow-up, introducing some risk of selection and attrition bias, respectively. Reporting bias across all studies was reduced due to an adequate description of the study re- sults. Similarly, the risk of detection and performance bias was minimal. The individual study and overall bias summaries are reported in Figures 1 and 2.

\section{Results}

A systematic search of the literature identified 1,371 articles. Following the removal of irrelevant and duplicate items, 80 articles were deemed relevant for full text review. We further excluded 71 articles based on selection criteria; nine clinical trials qualified for final analysis (Table 1) [6, 8-15]. All included trials enrolled patients undergoing $\mathrm{CABG}$ or having ACS or heart failure. The preferred reporting items for systematic reviews and meta-analyses (PRISMA) flow diagram is shown in Figure 3.

A total of 850 patients were included; 480 in the allopurinol and 370 in the placebo or control group. The mean age was 63.4 years, comprising $79 \%$ male patients. Of the total population, $84 \%$ of patients had a history of hypertension and $32 \%$ had diabetes mellitus. There was no significant difference in the baseline characteristics of the two groups across most of the studies. A significant amount of heterogeneity was observed in the selection criteria and follow-up durations of the included studies. Six of the included studies compared the utility of allopurinol in CABG patients, two trials studied the efficacy of allopurinol in the secondary prevention of ACS and one randomized controlled trial (RCT) studied the effect of allopurinol on heart failure-related hospitalizations. Huang et al [9] included patients after an acute event of ACS, while Goicoechea et al [8] recruited patients with chronic kidney disease (CKD). The follow-up period in patients undergoing $\mathrm{CABG}$ was up to 30 days post-procedure, while it was 2 years in studies on ACS and 24 weeks in the study on heart failure patients. The daily dose of allopurinol ranged from $100 \mathrm{mg}$ daily (for CKD patients in Goicoechea's study) to $600 \mathrm{mg}$ daily or 300 $\mathrm{mg}$ twice a day. The detailed baseline characteristics are given in Table $1[6,8-15]$.

\section{Allopurinol for periprocedural in CABG patients}

Five studies comprising 215 patients (allopurinol 106, control 109 ) contributed to this comparison. Patients receiving allopurinol during the $\mathrm{CABG}$ procedure had significantly lower odds 


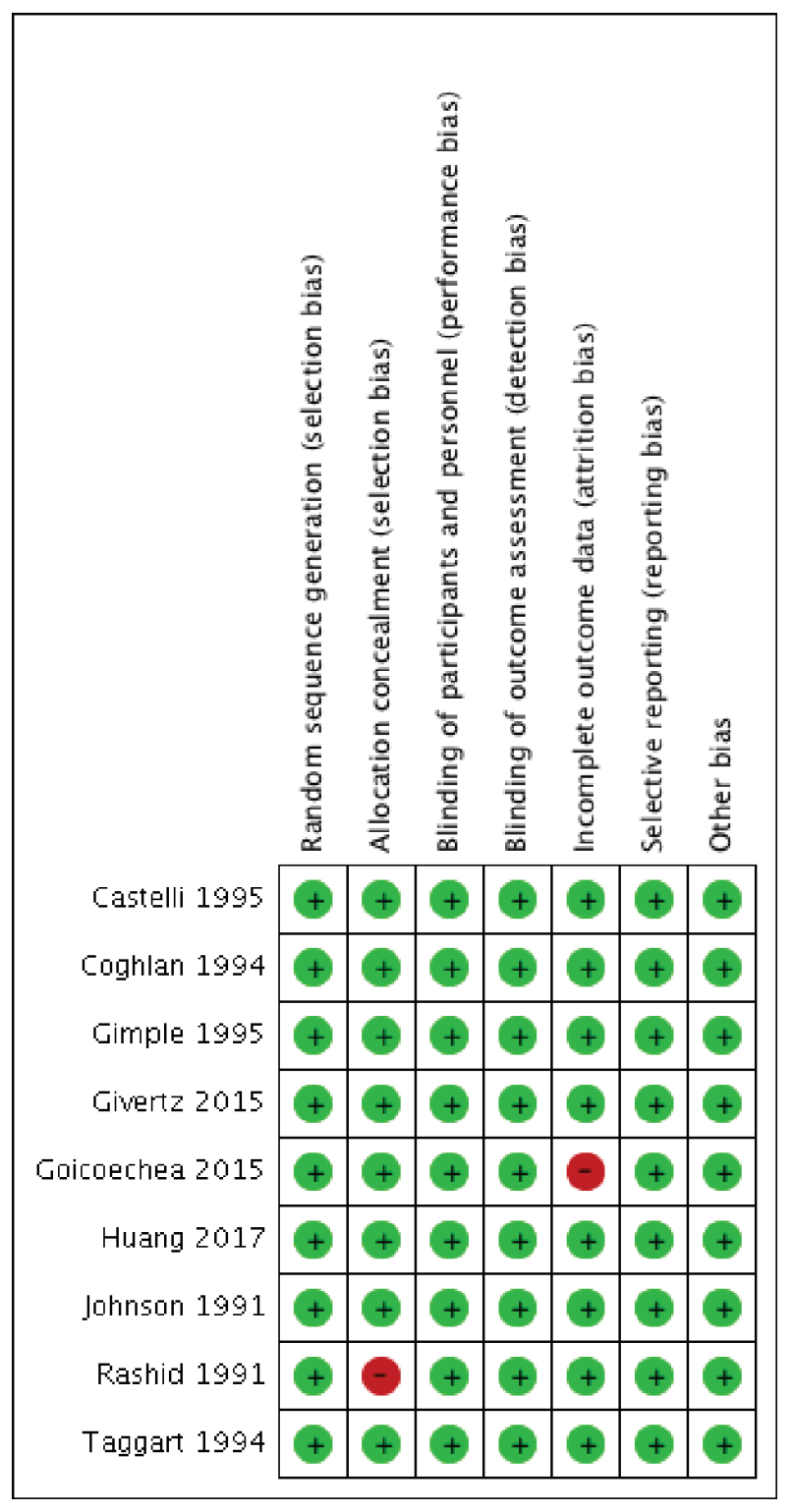

Figure 2. Detailed methodological quality assessment of the included studies showing minimal risk of bias (red).

of periprocedural ACS events by $75 \%$ compared to patients on placebo or no medication (OR: $0.25,95 \%$ CI: $0.06-0.96, \mathrm{P}=$ 0.05) (Fig. 1). On Higgin's equation, there was no heterogeneity in the outcomes of the included studies $\left(\mathrm{I}^{2}=0 \%\right)$ (Fig. 2). The study by Johnson et al [6] also showed that allopurinol could significantly decrease the odds of periprocedural cardiovascular mortality (OR: $0.22,95 \% \mathrm{CI}: 0.07-0.71, \mathrm{P}=0.01$ ) (Fig. 4).

A sensitivity analysis of periprocedural CABG-related ACS events based on the exclusion of the study by Rashid et al [10] showed no significant difference between two groups (OR: $0.37,95 \%$ CI: $0.10-1.32, \mathrm{P}=0.13$ ). This indicates that although all studies showed a numerical advantage of lower ACS events in the allopurinol group, the statistically beneficial findings were actually driven by one study.

\section{Allopurinol for secondary prevention}

The odds of cardiovascular mortality (OR: $0.33,95 \%$ CI: 0.01 - 8.21, P $=0.50$ ) or recurrent ACS events (OR: $0.32,95 \% \mathrm{CI}$ : $0.03-3.18, \mathrm{P}=0.33$ ) in the allopurinol group were not significantly different from the control group. Similarly, in patients with a diagnosed heart failure, the use of allopurinol for 24 weeks offered no benefits in terms of lower ACS events (OR: 1.12, 95\% CI: 0.39 - 3.20, $\mathrm{P}=0.83$ ) (Fig. 5).

\section{Publication bias}

The funnel plot showed asymmetry, indicating the possibility of publication bias. One study has shown the graphical violation of the midline, as depicted in Figure 6. One can argue that it is difficult to differentiate between "findings by chance" and "real asymmetry," as only six articles were assessed for potential publication bias. As pointed by Sterne et al in a study of fewer than 10 articles, it is difficult to differentiate between real asymmetry and findings merely by chance [16].

\section{Discussion}

Our results revealed that prophylactic use of allopurinol in patients undergoing CABG could potentially decrease periprocedural MI and cardiovascular mortality. These findings, however, should be interpreted with caution, given that they were driven only by one study (Rashid et al) [10]. On sensitivity analysis, there was no significant difference in the periprocedural MI event rate between patients receiving allopurinol and those on the placebo. Similarly, Johnson et al was the only study to demonstrate a significantly lower rate of periprocedural mortality in CABG patients on allopurinol [6]. Other studies compared the long-term efficacy of allopurinol in patients with CKD, ACS and congestive heart failure (CHF). The risk of recurrent $\mathrm{MI}$ and cardiovascular mortality at an extended follow-up duration of 2 years was found to be identical between patients in allopurinol and control groups $[8,9,11]$.

On review, we found a significant amount of heterogeneity in the inclusion criteria and outcomes of the included studies. Gimpel et al recruited 22 CABG patients (control 14, allopurinol eight) to determine the possibility of myocardial protection against reperfusion injury by allopurinol [14]. On a multivariate analysis adjusted for potential effect modifiers, there was no difference recorded between the two groups. This study, however, was designed to calculate the effect of the inflammatory markers (leukotrienes), and was underpowered to gauge the hard clinical outcomes (MI and mortality). By contrast, Rashid et al included $90 \mathrm{CABG}$ patients, half of which (n $=45$ ) received a loading dose of allopurinol in the perioperative period. Compared to the control arm, allopurinol had an $18 \%$ lower risk of perioperative MI. This numerical difference, however, did not reach the level of statistical significance [10].

Taggart et al randomized 20 patients to receive allopurinol or to act as controls [12]. The primary endpoint measured was the efficacy of myocardial protection determined by serial 


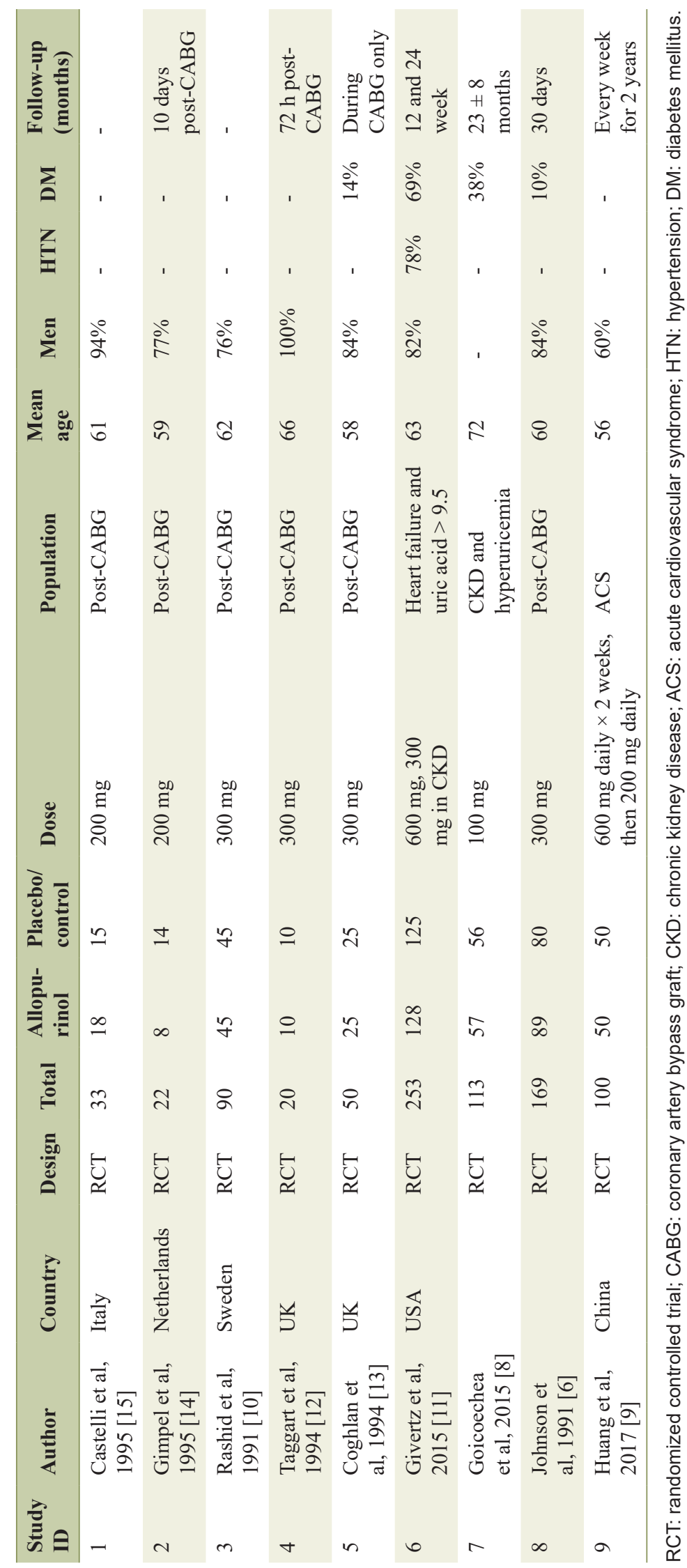




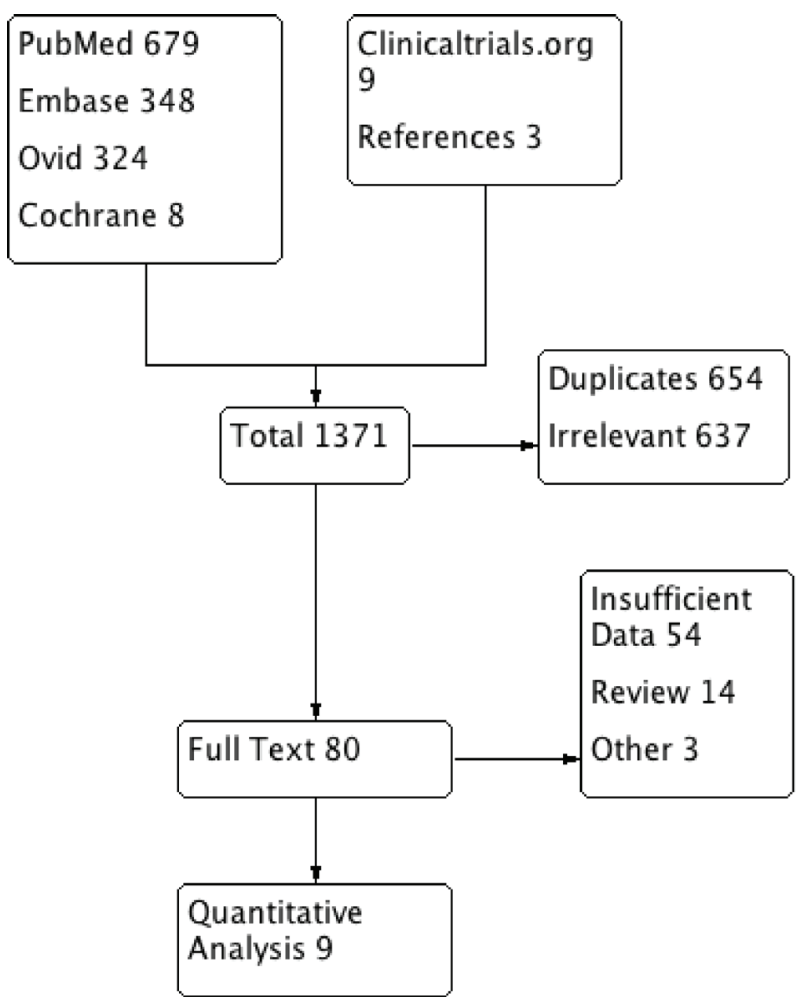

Figure 3. PRISMA flow diagram of the included studies. PRISMA: preferred reporting items for systematic reviews and meta-analyses.

measurement of high sensitive cardiac troponin $\mathrm{T}$ (cTnT), creatine kinase (CK)-MB and myoglobin. This study also demonstrated no significant difference in the levels of pre- and postoperative cardiac biomarkers or electrocardiogram (ECG) (no evidence of perioperative infarction) at 1, 6, 24, and $72 \mathrm{~h}$ after $\mathrm{CABG}$ procedure [12]. Coghlan et al subsequently showed that compared to the control group, allopurinol pretreatment in CABG patients could significantly reduce the use of inotropic support $(\mathrm{n}=5 / 25$ vs. $13 / 25, \mathrm{P} \leq 0.01)$ and can increase the cardiac index $(\mathrm{P} \leq 0.004)$ after the surgery [13]. These differences, however, did not translate into the clinical outcomes. Similar to the study of Taggart et al [12], an identical periprocedural MI rate was observed between the two groups [13]. Unfortunately, the comparison groups in all these studies were far from homogeneous, and the procedures performed were by different surgeons. Similarly, the duration of an ischemic event, degree of myocardial impairment and number of diseased coronary vessels were not consistent.

Johnson et al hurdled these limitations by recruiting 169 patients (allopurinol 89, placebo 80), matched for their surgical risk factors and baseline comorbidities [6]. Contrary to previous studies, the in-hospital mortality rate in the allopurinol group $(n=4 / 89,4 \%)$ was significantly lower than the placebo group $(\mathrm{n}=14 / 80,18 \%, \mathrm{P}=0.014)$. Similarly, cardiac performance, scored by cardiac index and the need for mechanical and inotropic support, significantly favored the allopurinol group [6].

Three studies compared the utility of allopurinol for the secondary prevention of ACS and cardiovascular mortality. Huang et al recruited 100 patients (50 in each group) with the diagnosis of the ACS. At 1 month, the total effective rate of angina pectoris and ECG in the allopurinol group patients was $93 \%$ and $96 \%$, significantly better than the placebo arm $(76 \%$ and $82 \%$ ), respectively. The number of patients with stent implantation and cardiovascular mortality was not significantly different between the two groups at 2 years [9]. Givertz et al randomized 253 patients with symptomatic heart failure (ejection fraction less than $40 \%$ ) into allopurinol and a placebo group [11]. At 24 weeks, there was no significant difference in the clinical status of cardiomyopathy and ejection fraction between the allopurinol- and placebo-treated patients. The number of MI events was also identical between the two groups. This study could not exclude the possibility that a study on higher dose of allopurinol or long-term follow-up of a more homogeneous group of high-risk patients would have demonstrated significant reductions in heart failure-related mortality [11]. In the RCT by Goicoechea et al, 113 patients (allopurinol 57, control 56) with CKD (estimated glomerular filtration rate (eGFR) $<60 \mathrm{~mL} / \mathrm{min}$ ) were included [8]. Allopurinol treatment slowed down kidney disease progression but showed no significant difference in the 2-year rate of cardiovascular mortality. Other notable cardiovascular events in the allopurinol and control groups included heart failure in eight patients, ischemic coronary events, cerebrovascular accidents, peripheral arteriopathy, and arrhythmias in seven, five, one and one patients, respectively [8].

The present study systematically stratified patients based on their patient's inclusion criteria and follow-up duration. Briefly, allopurinol confers advantages over placebo if used in conjunction with cardioplegic therapy during the $\mathrm{CABG}$

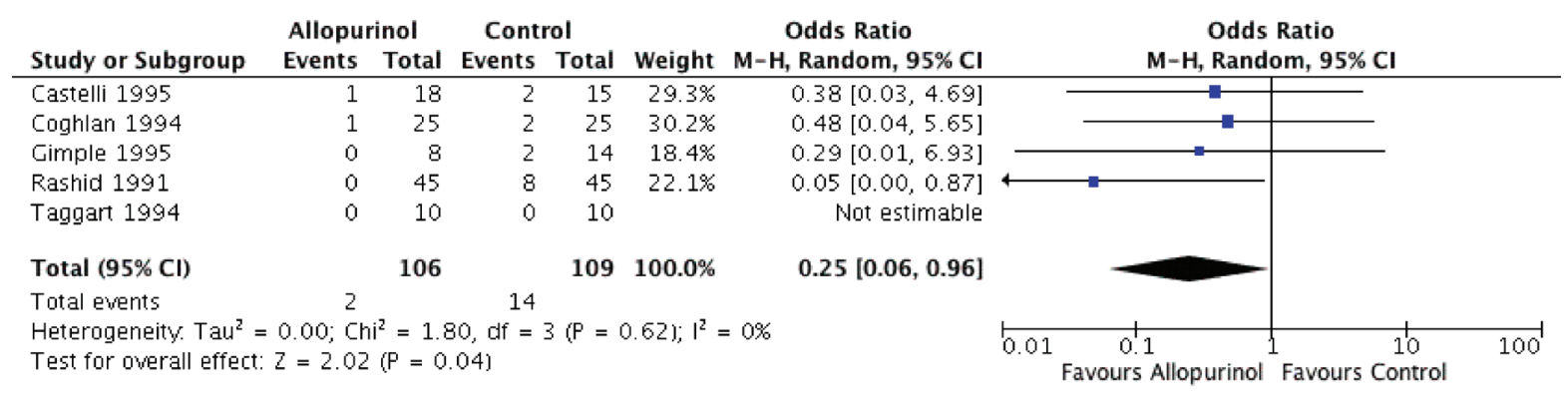

Figure 4. Forest plot of CABG patients showing significantly lower odds of periprocedural myocardial infarction in the allopurinol group compared to the control group. CABG: coronary artery bypass graft. 


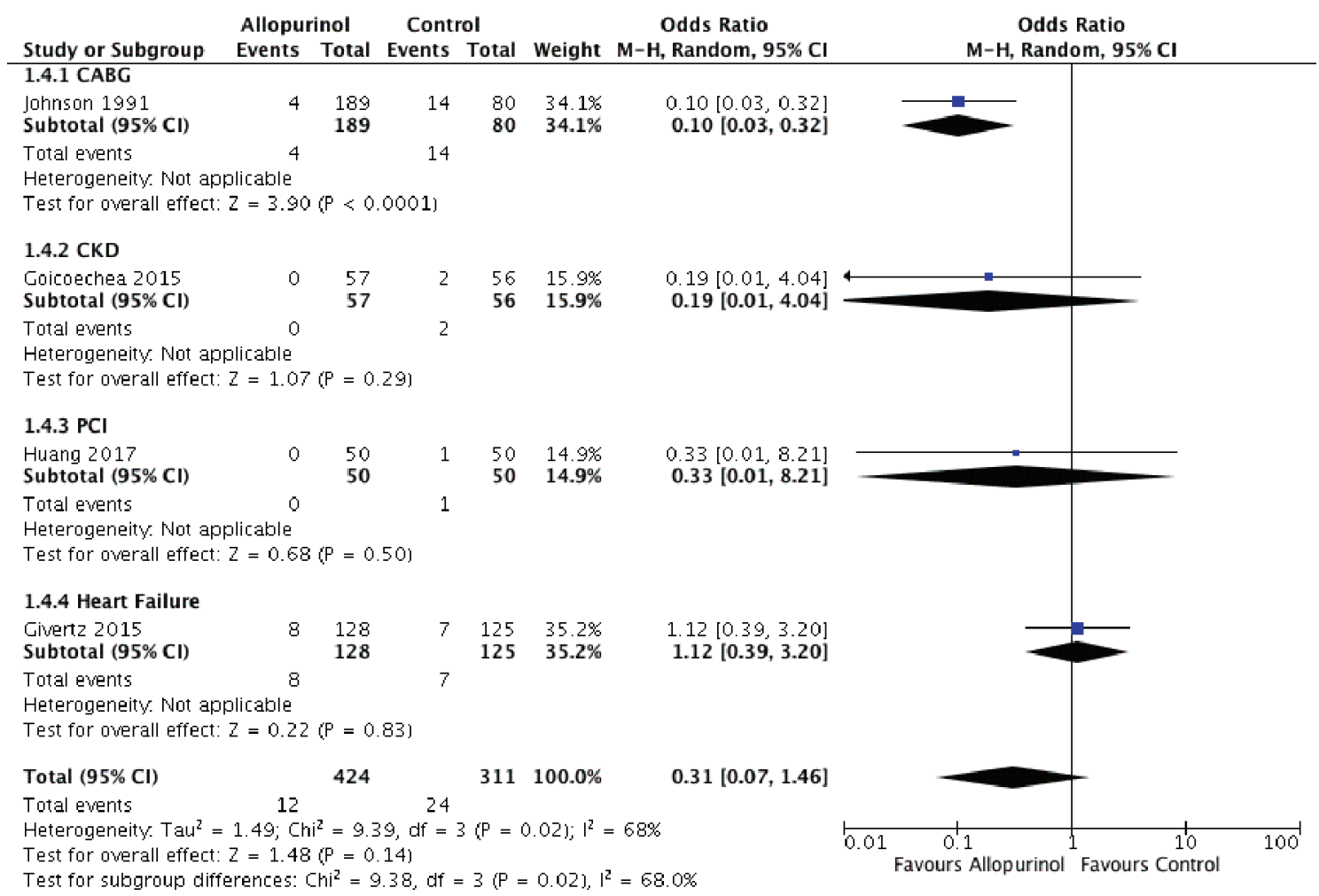

Figure 5. Forest plot showing lower CABG related mortality and an identical odds of cardiovascular mortality in the allopurinol group compared to the control group in patients with heart failure, CKD and after PCI. CABG: coronary artery bypass graft; CKD: chronic kidney disease; PCl: percutaneous coronary intervention; 95\% Cl: 95\% confidence interval.

procedure. Our study demonstrates that periprocedural MI and cardiovascular mortality might be substantially lower with the use of anti-free radical therapy. The long-term benefits of allopurinol could not be established in patients with a prior history of ACS, CKD, or heart failure.

\section{Limitations}

This meta-analysis was constrained by the limitations of the

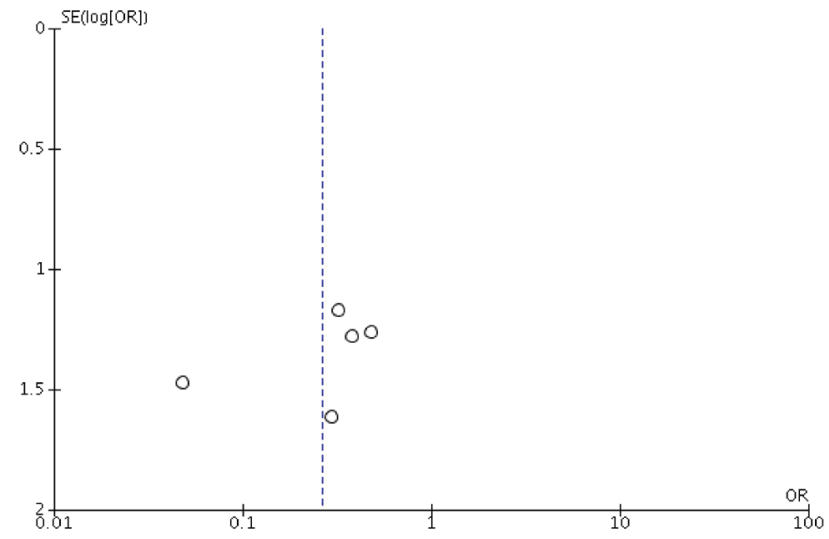

Figure 6. Funnel plot showing a possibility of publication bias or findings merely by chance. included studies. Only seven underpowered studies were included in the final analysis, indicating that our overall results were only modest in its statistical power. This, in addition to the vast heterogeneity of the inclusion criteria, calls for caution when interpreting the results of this meta-analysis. Patientlevel data were missing to determine the impact of baseline comorbidities on the overall clinical outcomes. The dose-response relationship and subgroup analysis based on the treatment vs. "intention-to-treat" group was lacking in most of the included studies, limiting our ability to calculate their predictive effects. Similarly, we could not account for the differential use of other prophylactic medications (in cases of CABG) or secondary preventive therapy (in post-ACS) patients between the two groups. All studies primarily focused on the biochemical outcomes, while hard clinical endpoints (mortality and MI) were recorded as secondary outcomes, potentially underpowering the overall results. The net clinical benefit of allopurinol could not be ascertained as a significant amount of allopurinolinduced complications such as renal and liver failure were neglected. This can potentially reduce the precision of our estimated results.

\section{Conclusions}

Allopurinol might potentially reduce the periprocedural adverse events, including MI and mortality in patients undergoing $\mathrm{CABG}$. However, it has no long-term benefits in terms of 
secondary prevention of ACS and mortality in patients with ACS, heart failure, or CKD. Larger scale studies are needed to determine the true merits of allopurinol in cardiovascular disease.

\section{Acknowledgments}

We sincerely thank Dr. Margot Boigon, Dr. David Smith and Dr. Richard Eisenstaedt for providing research opportunities and resources in the institute.

\section{Financial Disclosure}

None to declare.

\section{Conflict of Interest}

None to declare.

\section{Informed Consent}

Not applicable.

\section{Author Contributions}

Waqas Ullah: conceptualization, writing of the original draft, analysis, validation and visualization; Artem Minalyan: literature search; M. Chadi Alraies: critical review; Shristi Khanal: investigation; Rozi Khan: writing discussion; Bikash Basyal: data extraction; Samavia Munir: data extraction; David L. Fischman: supervision, review and editing.

\section{Data Availability}

Any inquiries regarding supporting data availability of this study should be directed to the corresponding author.

\section{References}

1. Bolli R, Hartley CJ, Chelly JE, Patel BS, Rabinovitz RS, Jeroudi MO, Roberts R, et al. An accurate, nontraumatic ultrasonic method to monitor myocardial wall thickening in patients undergoing cardiac surgery. J Am Coll Cardiol. 1990;15(5):1055-1065.

2. Bolli R, McCay PB. Use of spin traps in intact animals undergoing myocardial ischemia/reperfusion: a new approach to assessing the role of oxygen radicals in myocardial "stunning". Free Radic Res Commun. 1990;9(36):169-180.

3. Davies SW, Underwood SM, Wickens DG, Feneck RO,
Dormandy TL, Walesby RK. Systemic pattern of free radical generation during coronary bypass surgery. $\mathrm{Br}$ Heart J. 1990;64(4):236-240.

4. Ferreira R, Llesuy S, Milei J, Scordo D, Hourquebie H, Molteni L, de Palma C, et al. Assessment of myocardial oxidative stress in patients after myocardial revascularization. Am Heart J. 1988;115(2):307-312.

5. Cavarocchi NC, England MD, O'Brien JF, Solis E, Russo P, Schaff HV, Orszulak TA, et al. Superoxide generation during cardiopulmonary bypass: is there a role for vitamin E? J Surg Res. 1986;40(6):519-527.

6. Johnson WD, Kayser KL, Brenowitz JB, Saedi SF. A randomized controlled trial of allopurinol in coronary bypass surgery. Am Heart J. 1991;121(1 Pt 1):20-24.

7. Tabayashi K, Suzuki Y, Nagamine S, Ito Y, Sekino Y, Mohri H. A clinical trial of allopurinol (Zyloric) for myocardial protection. J Thorac Cardiovasc Surg. 1991;101(4):713-718.

8. Goicoechea M, Garcia de Vinuesa S, Verdalles U, Verde E, Macias N, Santos A, Perez de Jose A, et al. Allopurinol and progression of CKD and cardiovascular events: longterm follow-up of a randomized clinical trial. Am J Kidney Dis. 2015;65(4):543-549.

9. Huang Y, Zhang C, Xu Z, Shen J, Zhang X, Du H, Zhang $\mathrm{K}$, et al. Clinical Study on efficacy of allopurinol in patients with acute coronary syndrome and its functional mechanism. Hellenic J Cardiol. 2017;58(5):360-365.

10. Rashid MA, William-Olsson G. Influence of allopurinol on cardiac complications in open heart operations. Ann Thorac Surg. 1991;52(1):127-130.

11. Givertz MM, Anstrom KJ, Redfield MM, Deswal A, Haddad H, Butler J, Tang WH, et al. Effects of xanthine oxidase inhibition in hyperuricemic heart failure patients: the xanthine oxidase inhibition for hyperuricemic heart failure patients (EXACT-HF) Study. Circulation. 2015;131(20):1763-1771.

12. Taggart DP, Young V, Hooper J, Kemp M, Walesby R, Magee P, Wright JE. Lack of cardioprotective efficacy of allopurinol in coronary artery surgery. Br Heart J. 1994;71(2):177-181.

13. Coghlan JG, Flitter WD, Clutton SM, Panda R, Daly R, Wright G, Ilsley CD, et al. Allopurinol pretreatment improves postoperative recovery and reduces lipid peroxidation in patients undergoing coronary artery bypass grafting. J Thorac Cardiovasc Surg. 1994;107(1):248-256.

14. Gimpel JA, Lahpor JR, van der Molen AJ, Damen J, Hitchcock JF. Reduction of reperfusion injury of human myocardium by allopurinol: a clinical study. Free Radic Biol Med. 1995;19(2):251-255.

15. Castelli P, Condemi AM, Brambillasca C, Fundaro P, Botta M, Lemma M, Vanelli P, et al. Improvement of cardiac function by allopurinol in patients undergoing cardiac surgery. J Cardiovasc Pharmacol. 1995;25(1):119-125.

16. Sterne JA, Sutton AJ, Ioannidis JP, Terrin N, Jones DR, Lau J, Carpenter J, et al. Recommendations for examining and interpreting funnel plot asymmetry in meta-analyses of randomised controlled trials. BMJ. 2011;343:d4002. 